\section{Comparison of subfoveal choroidal thickness in healthy pregnancy and pre-eclampsia}

\begin{abstract}
Purpose Pregnancy is a known predisposing factor for central serous chorioretinopathy (CSC). Choroidal thickness (CT) increases in patients with CSC. This study was designed to evaluate CT in pregnant women.

Patients and methods This was a prospective study. Fourteen healthy pregnant women and seven patients with pre-eclampsia were included. Twenty-one normal subjects were also recruited. CT was measured using enhanced-depth imaging optical coherence tomography.

Results The mean CT of normal subjects, healthy pregnant women and patients with pre-eclampsia were $264.95 \pm 21.03$, $274.23 \pm 29.30$ and $389.79 \pm 25.13 \mu \mathrm{m}$, respectively (normal subjects $v$ s healthy gravidas: $P>0.05$; normal subjects $v s$ pre-eclampsia: $P<0.001$; healthy gravidas $v s$ pre-eclampsia: $P<0.001)$. CT decreased from $381.05 \pm 22.96 \mu \mathrm{m}$ to $335.17 \pm 9.97 \mu \mathrm{m} 1$ week after delivery in patients with pre-eclampsia. Conclusions Pregnancy itself did not increase CT, whereas pre-eclampsia did appear to result in increased CT. This suggests that additional unknown factors induce hyperpermeability in pregnant women.

Eye (2016) 30, 349-354; doi:10.1038/eye.2015.215; published online 6 November 2015
\end{abstract}

\section{Introduction}

Pregnancy is considered a risk factor for central serous chorioretinopathy (CSC). The largest case-control study of patients with CSC found an odds ratio of 7.1 in women with prior or current pregnancy compared with their agematched counterparts with no history of pregnancy. ${ }^{1}$ However, it is unclear whether CSC is directly caused by pregnancy per se or is related to hormonal hypercoagulability or
JW Kim ${ }^{1}, \mathrm{MH}$ Park $^{2}, \mathrm{YJ} \mathrm{Kim}^{2}$ and YT Kim ${ }^{1,3}$

hemodynamic changes that occur as a result of pregnancy. ${ }^{2}$

Pre-eclampsia is a pregnancy-specific syndrome defined by hypertension and proteinuria that may also be associated with a myriad of other signs and symptoms, including oedema, visual disturbances, headache, and epigastric pain. ${ }^{3}$ Approximately $5 \%$ of obstetric patients develop pre-eclampsia with worldwide mortality rate and a high index of maternal and perinatal morbidity. ${ }^{4}$ The visual system is affected, with symptoms in $25-50 \%$ of patients with pre-eclampsia. ${ }^{5}$

Choroidal vascular hyperpermeability is a major pathophysiology of CSC. However, the use of fluorescein or indocyanine green angiography in pregnant women is limited, because of their teratogenic effects and concerns regarding breast-feeding. Currently, optical coherence tomography with an adaptation of its software for enhanced-depth imaging is expected to enable non-invasive evaluation of the choroid. In a previous study, choroidal thickening with choroidal vascular dilatation was observed in patients with CSC. ${ }^{6}$ Thus, we compared choroidal thickness (CT) between pregnant and non-pregnant women using enhanced-depth imaging optical coherence tomography. In addition, CT in pre-eclamptic patients was compared with that in normal subjects and healthy pregnant women.

\section{Materials and methods}

This study was conducted to compare subfoveal choroidal thickness (SFCT) and central subfield retinal thickness (CSRT) of 21 eyes of 21 pregnant women (study group) and 21 eyes of 21 normal subjects (control group). All pregnant women were recruited from the Department of Gynecology and Obstetrics at the same university, whereas normal subjects were
${ }^{1}$ Department of Ophthalmology, Ewha Womans University, School of Medicine, Seoul, South Korea

${ }^{2}$ Department of Obstetrics and Gynecology, Ewha Womans University, School of Medicine, Seoul, Korea

${ }^{3}$ Global Top 5 Research Program, Ewha Womans University, School of Medicine, Seoul, Korea

Correspondence: YT Kim, Department of Ophthalmology, Ewha Womans University Mok-dong Hospital, Mok-dong Yangcheon-ku, Seoul, 158-710, Korea Tel +82 226505154 ; Fax +82 226504334 . E-mail: jjongofhim@ hanmail.net

Received: 9 October 2014 Accepted in revised form: 12 September 2015 Published online: 6 November 2015 
recruited from the Ophthalmology Clinic.

This prospective study was conducted under institutional review board approval and all subjects were consented to participate in study.

Inclusion criteria for pregnant women were: a single pregnancy in the third trimester and no refractive errors $>-6.0$ or +6.0 D. Pre-eclamptic women, as established by the National High Blood Pressure Education Program criteria, were included in the study group. ${ }^{3}$ Pre-eclampsia was determined by increased blood pressure (BP) accompanied by proteinuria after the 20th week of pregnancy. Gestational BP elevation was defined as a $\mathrm{BP}>140 \mathrm{~mm} \mathrm{Hg}$ systolic or $>90 \mathrm{~mm} \mathrm{Hg}$ diastolic in a woman who was normotensive prior to 20 weeks of gestation. Inclusion criteria for normal subjects were: childbearing age (20-40 years); no reproductive history; and a normal ophthalmologic exam. Exclusion criteria were: smoking; drug abuse; significant cardiac disease; systemic diseases, including chronic systemic hypertension/diabetes mellitus; noticeable eye disorders, such as glaucoma or retinopathy; or previous ocular surgery, including refractive surgeries.

All subjects underwent full ophthalmic examinations, including a visual acuity test, complete slit-lamp examination of the anterior and posterior segments, and spectral domain optical coherence tomography (Spectralis OCT, Heidelberg, Germany). To obtain images of the choroid, we adopted an enhanced-depth imaging mode, which was provided by the manufacturer (Heidelberg Engineering, Heidelberg, Germany). Transfoveal horizontal and vertical line sections, each composed of 100 averaged scans were obtained for measuring SFCT. Employing the manual caliper provided with the intrinsic Heidelberg software, SFCT was measured at the centre. SFCT was defined as the vertical distance from the hyperreflective line of Bruch's membrane to chorio-scleral junction. When the chorio-scleral interface was not visible on the thick choroid, the outermost hyporeflective area of the choroid was measured. ${ }^{7}$ Two SFCT measurements from a horizontal scan and a vertical scan were averaged, and those averaged values were used for statistical analysis. ${ }^{8}$ The 6 -mm macular map was used to evaluate the central subfield retinal thickness in $1 \mathrm{~mm}$ of macular map.

The SPSS software version 18.0 for Windows (SPSS Inc., Chicago, IL, USA) was used for statistical analysis. Because the number of subjects was small, Kruskal-Wallis test was used to compare the age and spherical equivalent SFCT, and CRT in eyes of pre-eclamptic patients, healthy pregnant women, and normal subjects. In addition, for post hoc analysis, Bonferroni correction was used to compare SFCT among the three groups. For the comparisons of age and spherical equivalent, SFCT and CST in eyes of pregnant women with those of normal eyes, the Mann-Whitney $U$-test was used. The change in the CT measurements after delivery in pre-eclamptic patients was analysed using Wilcoxon's signed-rank test. Results are as means \pm SD and were considered to be statistically significant at $P<0.05$. For the power calculation of this study, post hoc power analysis (for $P$-value of $<0.05$, total sample size of 42 for a two-tailed hypothesis) demonstrated an observed power of 0.889 .

\section{Results}

The study group included 14 in the healthy pregnancy group and seven in the pre-eclampsia group, whereas the control group consisted of 21 healthy non-

pregnant women.

There was no significant difference in mean age, spherical equivalent or CSRT among the three groups ( $P=0.454,0.789$, and 0.722 , respectively). In addition, no significant difference in the gestational age between the healthy pregnancy and pre-eclampsia groups was found $(P=0.128$, Table 1). SFCT in normal subjects, the healthy pregnancy group and the pre-eclampsia group were $264.95 \pm 21.03,274.23 \pm 29.30$, and $389.79 \pm 25.13 \mu \mathrm{m}$, respectively; the differences among the three groups were significant $(P<0.001)$. Post hoc analysis revealed that SFCT of the healthy pregnancy group showed no significant difference from that of control group $(P=0.325)$, whereas SFCT of the pre-eclampsia group was

Table 1 Comparisons among the normal subjects, healthy pregnant women and pre-eclamptic women

\begin{tabular}{lcccc}
\hline & Normal & Healthy pregnancy & Pre-eclampsia & P-value \\
\hline Number & 21 & 14 & 7 & $0.454^{\mathrm{a}}$ \\
Age (years) & $30.43 \pm 4.15$ & $31.43 \pm 2.88$ & $31.86 \pm 4.06$ & $0.789^{\mathrm{a}}$ \\
SE (D) & $-1.88 \pm 1.62$ & $-1.36 \pm 2.60$ & $-2.04 \pm 1.88$ & $0.128^{\mathrm{b}}$ \\
Gestational age (weeks) & & $32.14 \pm 2.69$ & $34.57 \pm 16.81$ & $0.000^{\mathrm{a}}$ \\
SFCT $(\mu \mathrm{m})$ & $264.95 \pm 21.03$ & $274.23 \pm 29.30$ & $389.79 \pm 25.13$ & $0.722^{\mathrm{a}}$ \\
CSRT $(\mu \mathrm{m})$ & $264.48 \pm 18.73$ & $258.93 \pm 18.97$ & $262.29 \pm 12.39$ & \\
\hline
\end{tabular}

Abbreviations: CSRT, central subfield retinal thickness; SE, spherical equivalent; SFCT, subfoveal choroidal thickness.

Data presented as means \pm SD. ${ }^{a}$ Kruskal-Wallis test. ${ }^{b}$ Mann-Whitney test. 

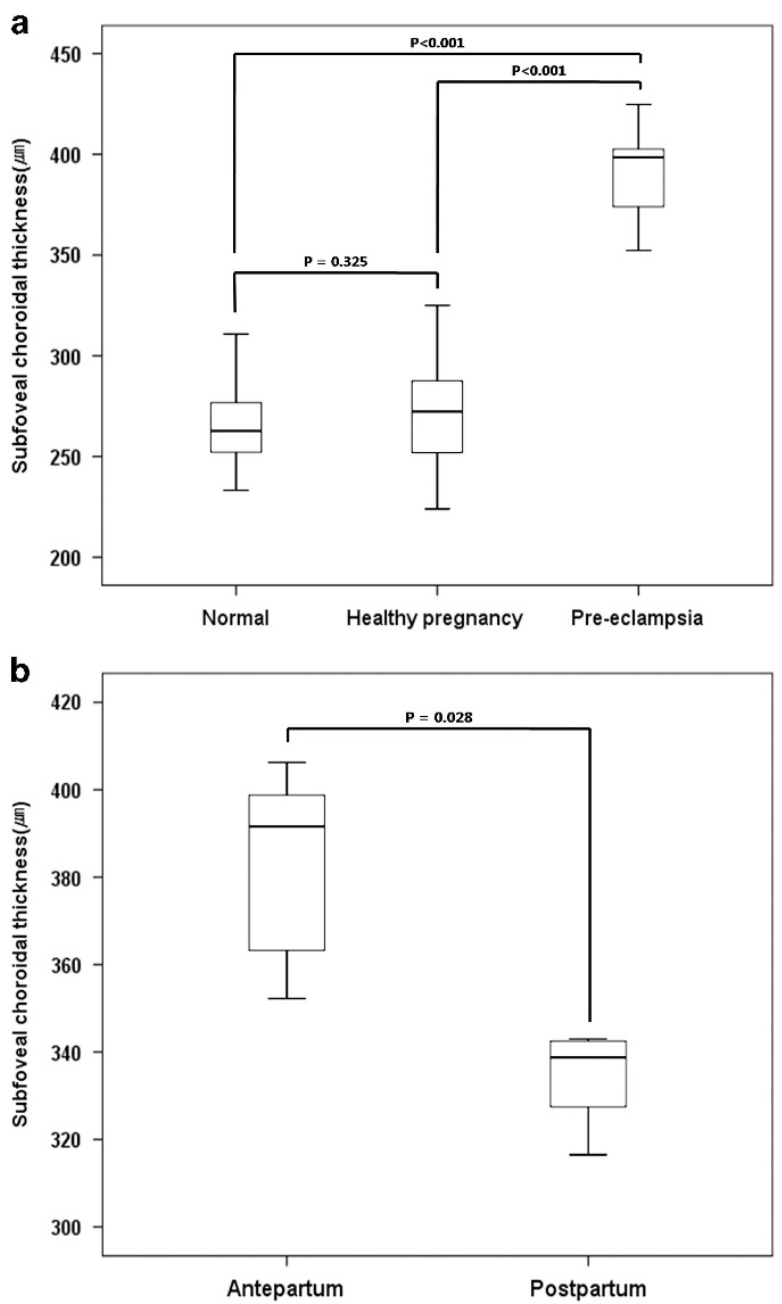

Figure 1 (a) Subfoveal choroidal thicknesses of pre-eclampsia, healthy pregnancy, and control subjects. There was no significant difference between normal subjects and healthy pregnant women $(P=0.325)$. However, SFCT in pre-eclamptic patients was significantly greater than that in normal subjects $(P<0.001)$ and healthy pregnant women $(P<0.001)$. (b) Change in the subfoveal choroidal thickness of pre-eclamptic women. There was a significant decrease from $383.96 \pm 8.87 \mu \mathrm{m}$ antepartum to $335.17 \pm 9.97 \mu \mathrm{m}$ at 1 week after delivery $(P=0.028)$.

significantly greater than in the control group $(P<0.001)$ and the healthy pregnancy group $(P<0.001)$ (Figure $1 \mathrm{a})$.

Of the seven eyes with pre-eclampsia, only six underwent repeat optical coherence tomography 1 week after delivery. Changes in SFCT in the pre-eclampsia group are summarised in Table 2 . The BP of pre-eclamptic patients in this study was $>140 / 100 \mathrm{~mm} \mathrm{Hg}$ at the time of diagnosis of pre-eclampsia. All patients were treated with hypotensive medication. After treatment, BP of pre-eclamptic patients were $<140 / 100 \mathrm{~mm} \mathrm{Hg}$, with one exception, at the antepartum examination. In the pre-eclampsia group, SFCT decreased significantly from $381.05 \pm 22.96 \mu \mathrm{m}$ (range, 352.25-406.25 $\mu \mathrm{m}$ ) at antepartum to $335.17 \pm 9.97 \mu \mathrm{m}$ (range, 318.75-343.50 $\mu \mathrm{m}$ ) after delivery $(P=0.028$, Figure $1 b)$.

\section{Discussion}

Previous observations of choroidal vasculature using enhanced-depth imaging optical coherence tomography demonstrated changes in CT in various retinal diseases, including age-related macular degeneration, CSC, and polypoidal choroidal vasculopathy. ${ }^{9-11}$ Choroidal thickening was observed in eyes with CSC or polypoidal choroidal vasculopathy and choroidal atrophy was also noted in eyes with age-related macular degeneration. 6,12,13 An association between choroidal thickening and choroidal vascular hyperpermeability was reported in previous studies. ${ }^{10,14}$

The cause of CSC development in pregnant women is not fully understood, and angiographic study is limited with respect to possible transplacental transport or lactation. Enhanced-depth imaging optical coherence tomography enables non-invasive and non-toxic assessment of choroidal vasculature. Here, we hypothesised that the choroid would be thickened in the eyes of pregnant women, because pregnancy itself is considered a risk factor for CSC.

There was no significant difference in CT between the healthy pregnancy group and normal subjects and CT was significantly greater in only the pre-eclampsia group compared with normal subjects. This suggests that pregnancy per se does not increase permeability of the choroid; rather hyperpermeability of the choroid occurs in only some pregnant women.

In previous studies, CSC could occur in healthy pregnant women, for which hormonal hypercoagulability or hemodynamic change during pregnancy is thought to be responsible..$^{2,15,16}$ In this study, however, there was no significant increase in CT in healthy pregnant women. Thus, we suggest that unknown additional factors such as vascular hyperpermeability, increased body fluid, vascular dilation, decreased colloidal osmotic pressure and changes in prostaglandin levels induce hyperpermeability and lead to CSC in pregnant women.

Alterations in the choroidal circulation and choroidal ischaemia preceding retinal vascular abnormality in pre-eclampsia have been reported. ${ }^{17,18}$ Valluri et al ${ }^{19}$ using indocyanine green angiography in pre-eclampsia reported choroidal ischaemia and staining as well as hyperpermeability of choroidal vessels, which suggested severe damage to the choroidal vascular walls. In addition, Sathish et al ${ }^{18}$ reported serous retinal detachment secondary to choroidal ischaemia with delayed filling of the choriocapillaries in fluorescein angiograms. These findings are similar to the choroidal alterations in CSC. Hyperpermeability and increased 
choroidal pressure as well as a filling delay in the area corresponding to a retinal pigment epithelial detachment were also found in CSC. In addition, these findings in pre-eclampsia may or may not be associated with blood pressure. ${ }^{18} \mathrm{Few}$ studies have been performed on the relationship between hypertension and CT. Ahn et al20 reported an increase in subfoveal $\mathrm{CT}$ and the accumulation of subretinal fluid followed by a decrease after blood pressure control in their studies of the retinal and choroidal changes in severe hypertension patients. Iwase $e a^{21}$ reported that the relationship between choroidal thickness and systolic blood pressure showed diurnal variation. These studies found that $\mathrm{CT}$ increased with blood pressure. In this study, the pre-eclampsia group had blood pressure above 140/100 $\mathrm{mm} \mathrm{Hg}$ when diagnosed with pre-eclampsia, and all showed decreased blood pressure after BP control, except one patient, when CT was measured. Therefore, it is unclear whether the rapid increase or decrease of blood pressure influenced the CT. Pre-eclampsia is believed to be caused by several factors including genetic factor, oxidative stress, increased autoboantibodies against type1 angiotensin II receptor 1, and abnormal trophoblast/decidual interaction to have failure of trophoblastic invasion into spiral arterioles.
As a result, anti-angiogenesis is stimulated, whereas angiogenesis being inhibited to activate leucocytes and endothelial cells leading to the abnormal uteroplacental circulation and increase in resistance to flow within the uterine arteries. This placental dysfunction is not limited to one organ but also has systemic influences such as proteinuria, renal failure, pancreatitis, acute fatty liver, hypertension, cardiac failure, pulmonary oedema, haemolytic anaemia, thrombocytopenia, and intracranial haemorrhage. ${ }^{22}$ It could be inferred that choroidal thickening in the eyes of patients with pre-eclampsia may arise through an identical mechanism. In addition, this finding implies a correlation between the occurrence of CSC in pregnant women and increased CT in pre-eclamptic patients, because increased choroidal hydrostatic pressure contributes to CSC occurrence, and choroidal delayed filling in CSC was reported previously. $6,10,23$

In this study, a small amount of subretinal fluid was seen in one patient with pre-eclampsia, but it was absorbed after delivery. Note that the systemic hypertension in this patient was controlled 5 days before the ophthalmic examination, and the CT decreased with the disappearance of the subretinal fluid (Figure 2). As subretinal fluid was observed without systemic hypertension or hypertensive retinopathy,

Table 2 Change in the subfoveal choroidal thickness of pre-eclamptic women

\begin{tabular}{|c|c|c|c|c|c|c|c|}
\hline Number & Age (years) & $S E(D)$ & Antepartum ( $\mu m)$ & Postpartum $(\mu m)$ & $B P 1^{\mathrm{a}}(\mathrm{mm} \mathrm{Hg})$ & $B P 2^{\mathrm{a}}(m m H g)$ & \\
\hline 1 & 26 & -0.75 & 384.75 & 341.75 & $170 / 110$ & $124 / 72$ & Severe pre-eclampsia \\
\hline 2 & 29 & 0.25 & 398.50 & 318.75 & $163 / 115$ & $131 / 93$ & \\
\hline 3 & 34 & -3.75 & 352.25 & 336.50 & $160 / 107$ & $141 / 100$ & \\
\hline 4 & 31 & -2.50 & 363.25 & 327.75 & $187 / 123$ & $134 / 95$ & \\
\hline 5 & 30 & -0.50 & 406.25 & 343.50 & $160 / 100$ & $130 / 65$ & \\
\hline 6 & 38 & -5.00 & 398.75 & 342.75 & $147 / 104$ & $120 / 75$ & \\
\hline 7 & 35 & -2.00 & 424.75 & & $150 / 110$ & $110 / 70$ & Follow-up loss \\
\hline Total $(\mu \mathrm{m})^{\mathrm{b}}$ & & & $383.96 \pm 8.87$ & $335.17 \pm 9.97$ & & & $P=0.028^{c}$ \\
\hline
\end{tabular}

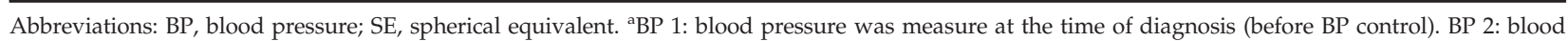
pressure was measured at antepartum ophthalmic examination (after BP control). ' Data are presented as means \pm SD. 'Wilcoxon's signed-rank test.
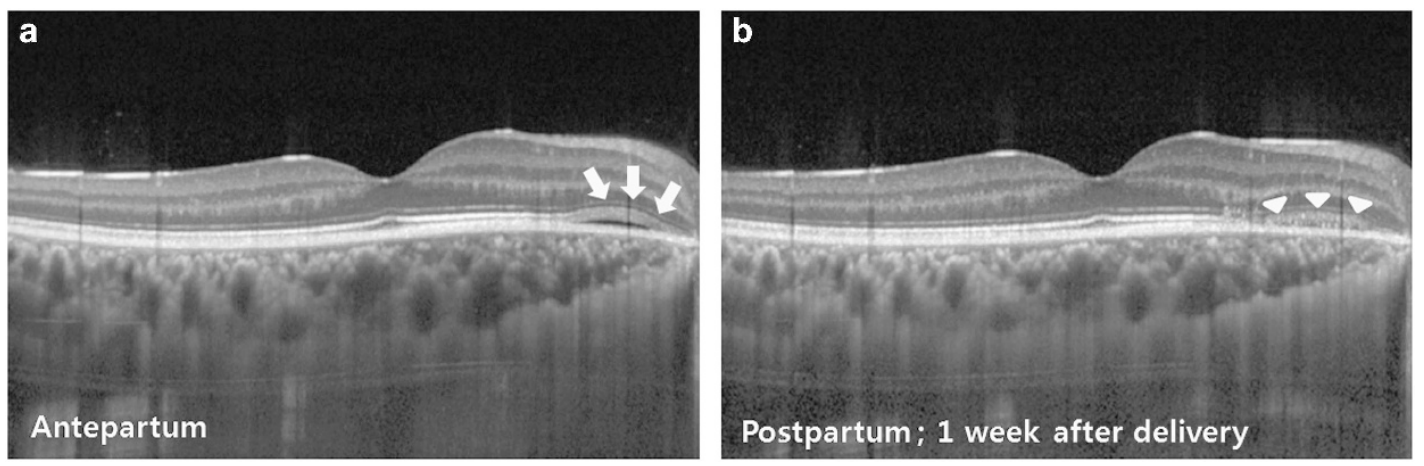

Figure 2 Enhanced-depth imaging OCT of a patient who showed spontaneous resolution of localised serous retinal detachment. Subfoveal choroidal thickness was $384.75 \mu \mathrm{m}$ with subretinal fluid (arrow) antepartum (a), and decreased to $341.75 \mu \mathrm{m}$ with retinal pigment epithelial change (arrowhead) at 1 week after delivery (b). 
the subretinal fluid may not have been due to the hypertensive retinopathy. Therefore, this finding suggests that subretinal fluid in the eyes in pre-eclampsia originates partly from increased hydrostatic pressure due to choroidal thickening.

Increased subfoveal CT could be associated with elevated intracranial pressure in pre-eclamptic/eclamptic patients. Previously, the association between subfoveal choroid and the cerebrospinal fluid pressure has been reported. ${ }^{24}$ Although it is difficult to measure the actual intracranial pressure in patients with pre-eclampsia/ eclampsia, ocular ultrasonography, and optic nerve sheath diameter measurement suggested that the incidence of increased intracranial pressure in preeclamptic patients would be $\sim 20 \%{ }^{25}$ Because the choroidal blood drains through the vortex veins and the superior orbital vein, which joins the intracranial cavernous sinus, increased intracranial pressure may have a role in increased choroidal thickness.

Using enhanced-depth imaging optical coherence tomography, Takahashi et $a^{26}$ also found no significant difference in CT between pregnant and control groups as seen in our study. By contrast, Sayin et $a l^{27}$ reported that the CT increased in a normal pregnant group compared with control and pre-eclampsia groups, with no significant difference between the control and pre-eclampsia groups. They explained this by an increase in cardiac output and ocular blood flow in the pregnant group to increase CT and systemic vascular resistance causing no increase in the CT, which was observed in the normal pregnant group. However, according to other studies, the choroidal blood flow changes constantly in the first and second trimesters, when measured by pulsatile ocular blood flow. ${ }^{28}$ In addition, gestational age should be considered in studies of pregnant women because systemic and ocular changes persist until the end of the pregnancy. ${ }^{28-31}$ In this study and Takahashi et al, the target subjects were limited to the last trimester, whereas Sayin et al included subjects in both the second and third trimesters, which may have caused the difference. Moreover, as mentioned previously, endothelial cell dysfunction and hyperpermeability are major causes of multi-organ failure in pre-eclampsia. ${ }^{22}$ As a result, cytotoxic and vasogenic oedema cause numerous changes in various organs, such as cerebral, pulmonary, and generalised oedema. Therefore, it is thought that the choroid may have increased interstitial oedema and CT in the same process as other organs. Finally, the target subjects with pre-eclampsia in this study were screened by examining patients with severe pre-eclampsia who required Caesarean sections before the planned birth date when they were hospitalised, which could have resulted in the more significant choroidal vascular hyperpermeability observed in pre-eclampsia vs that seen in previous studies.

In this study, there were no significant differences in CSRT among the three groups. Demir et $a l^{32}$ reported no significant difference in CSRT between 40 healthy pregnant women in the third trimester and 40 normal subjects. However, Cankaya et al ${ }^{33}$ showed that CSRT in the second and third trimester groups was significantly greater than in the first trimester and non-pregnant groups. In addition, they suggested that the increased volume of fluid in the body secondary to hormonal change may induce an increase in CSRT. This discrepancy was related to the fact that CSRT was defined as a retinal thickness of 1-mm diameter on a macular map by both Demir et $a l^{32}$ and by us, whereas it was defined as a retinal thickness of $3 \mathrm{~mm}$ diameter by Cankaya et al. ${ }^{33}$

A limitation of this study is the small number of subjects. In addition, the follow-up visit after delivery did not occur in many cases because of the inconvenience and disability after delivery. Another limitation was the relatively high prevalence of pre-eclampsia. As this study was conducted in a tertiary hospital, the prevalence of pre-eclampsia in the study group was too high to be generalised.

Although systemic changes in pre-eclampsia patients such as increase in vascular resistance are alleged, little is known about changes in choroid and retina. This study suggests that eclampsia may increase CT, although pregnancy itself does not.

\section{Summary}

What was known before

- Pregnancy is a known predisposing factor for CSC. CT increases in patients with CSC.

- The change of CT in pregnant women is not yet known clearly.

What this study adds

- Pregnancy itself did not increase CT, whereas pre-eclampsia did appear to result in increased CT.

\section{Conflict of interest}

The authors declare no conflict of interest.

\section{Acknowledgements}

This research was supported by Basic Science Research Program through the National Research Foundation of Korea (NRF) funded by the Ministry of Education, Science and Technology (2013R1A1A1060545). 


\section{References}

1 Haimovici R, Koh S, Gagnon DR, Lehrfeld T, Wellik S. Risk factors for central serous chorioretinopathy: a case-control study. Ophthalmology 2004; 111(2): 244-249.

2 Sunness JS, Haller JA, Fine SL. Central serous chorioretinopathy and pregnancy. Arch Ophthalmol 1993; 111(3): 360-364.

3 Sibai B, Dekker G, Kupferminc M. Pre-eclampsia. Lancet 2005; 365(9461): 785-799.

4 Sheth BP, Mieler WF. Ocular complications of pregnancy. Curr Opin Ophthalmol 2001; 12(6): 455-463.

5 Schultz KL, Birnbaum AD, Goldstein DA. Ocular disease in pregnancy. Curr Opin Ophthalmol 2005; 16(5): 308-314.

6 Imamura Y, Fujiwara T, Margolis R, Spaide RF. Enhanced depth imaging optical coherence tomography of the choroid in central serous chorioretinopathy. Retina 2009; 29(10): 1469-1473.

7 Spaide RF, Koizumi H, Pozzoni MC. Enhanced depth imaging spectral-domain optical coherence tomography. Am J Ophthalmol 2008; 146(4): 496-500.

8 Cho AR, Choi YJ, Kim YT. Influence of choroidal thickness on subfoveal choroidal thickness measurement repeatability using enhanced depth imaging optical coherence tomography. Eye (Lond) 2014; 28(10): 1151-1160.

9 Jirarattanasopa P, Ooto S, Nakata I, Tsujikawa A, Yamashiro K, Oishi A et al. Choroidal thickness, vascular hyperpermeability, and complement factor $\mathrm{H}$ in age-related macular degeneration and polypoidal choroidal vasculopathy. Invest Ophthalmol Vis Sci 2012; 53(7): 3663-3672.

10 Kuroda S, Ikuno Y, Yasuno Y, Nakai K, Usui S, Sawa M et al. Choroidal thickness in central serous chorioretinopathy. Retina 2013; 33(2): 302-308.

11 Switzer DW Jr, Mendonca LS, Saito M, Zweifel SA, Spaide RF. Segregation of ophthalmoscopic characteristics according to choroidal thickness in patients with early age-related macular degeneration. Retina 2012; 32(7): 1265-1271.

12 Kim SW, Oh J, Kwon SS, Yoo J, Huh K. Comparison of choroidal thickness among patients with healthy eyes, early age-related maculopathy, neovascular age-related macular degeneration, central serous chorioretinopathy, and polypoidal choroidal vasculopathy. Retina 2011; 31(9): 1904-1911.

13 Spaide RF. Age-related choroidal atrophy. Am J Ophthalmol 2009; 147(5): 801-810.

14 Kim YT, Kang SW, Bai KH. Choroidal thickness in both eyes of patients with unilaterally active central serous chorioretinopathy. Eye (Lond) 2011; 25(12): 1635-1640.

15 Said-Ahmed K, Moustafa G, Fawzy M. Incidence and natural course of symptomatic central serous chorioretinopathy in pregnant women in a maternity hospital in Kuwait. Middle East Afr J Ophthalmol 2012; 19(3): 273-276.

16 Gass JD, Little H. Bilateral bullous exudative retinal detachment complicating idiopathic central serous chorioretinopathy during systemic corticosteroid therapy. Ophthalmology 1995; 102(5): 737-747.
17 Iida T, Kishi S. Choroidal vascular abnormalities in preeclampsia. Arch Ophthalmol 2002; 120(10): 1406-1407.

18 Sathish S, Arnold JJ. Bilateral choroidal ischaemia and serous retinal detachment in pre-eclampsia. Clin Experiment Ophthalmol 2000; 28(5): 387-390.

19 Valluri S, Adelberg DA, Curtis RS, Olk RJ. Diagnostic indocyanine green angiography in preeclampsia. Am J Ophthalmol 1996; 122(5): 672-677.

20 Ahn SJ, Woo SJ, Park KH. Retinal and choroidal changes with severe hypertension and their association with visual outcome. Invest Ophthalmol Vis Sci 2014; 55(12): 7775-7785.

21 Iwase T, Yamamoto K, Ra E, Murotani K, Matsui S, Terasaki H. Diurnal variations in blood flow at optic nerve head and choroid in healthy eyes: diurnal variations in blood flow. Medicine (Baltimore) 2015; 94(6): e519.

22 Chaiworapongsa T, Chaemsaithong P, Yeo L, Romero R. Pre-eclampsia part 1: current understanding of its pathophysiology. Nat Rev Nephrol 2014; 10(8): 466-480.

23 Guyer DR, Yannuzzi LA, Slakter JS, Sorenson JA, Ho A, Orlock D. Digital indocyanine green videoangiography of central serous chorioretinopathy. Arch Ophthalmol 1994; 112(8): 1057-1062.

24 Jonas JB, Wang N, Wang YX, You QS, Yang D, Xie X et al. Subfoveal choroidal thickness and cerebrospinal fluid pressure: the Beijing Eye Study 2011. Invest Ophthalmol Vis Sci 2014; 55(3): 1292-1298.

25 Dubost C, Le Gouez A, Jouffroy V, Roger-Christoph S, Benhamou D, Mercier FJ et al. Optic nerve sheath diameter used as ultrasonographic assessment of the incidence of raised intracranial pressure in preeclampsia: a pilot study. Anesthesiology 2012; 116(5): 1066-1071.

26 Takahashi J, Kado M, Mizumoto K, Igarashi S, Kojo T. Choroidal thickness in pregnant women measured by enhanced depth imaging optical coherence tomography. Jpn J Ophthalmol 2013; 57(5): 435-439.

27 Sayin N, Kara N, Pirhan D, Vural A, Araz Ersan HB, Tekirdag AI et al. Subfoveal choroidal thickness in preeclampsia: comparison with normal pregnant and nonpregnant women. Semin Ophthalmol 2014; 29(1): 11-17.

28 Centofanti M, Migliardi R, Bonini S, Manni G, Bucci MG, Pesavento CB et al. Pulsatile ocular blood flow during pregnancy. Eur J Ophthalmol 2002; 12(4): 276-280.

29 Qureshi IA. Measurements of intraocular pressure throughout the pregnancy in Pakistani women. Chin Med Sci J 1997; 12(1): 53-56.

30 Robson SC, Hunter S, Boys RJ, Dunlop W. Serial study of factors influencing changes in cardiac output during human pregnancy. Am J Physiol 1989; 256(4 Pt 2): H1060-H1065.

31 Thornburg KL, Jacobson SL, Giraud GD, Morton MJ. Hemodynamic changes in pregnancy. Semin Perinatol 2000; 24(1): 11-14.

32 Demir M, Oba E, Can E, Odabasi M, Tiryaki S, Ozdal E et al. Foveal and parafoveal retinal thickness in healthy pregnant women in their last trimester. Clin Ophthalmol 2011; 5: 1397-1400.

33 Cankaya C, Bozkurt M, Ulutas O. Total macular volume and foveal retinal thickness alterations in healthy pregnant women. Semin Ophthalmol 2013; 28(2): 103-111. 